\title{
Seven-day intensive cognitive therapy for PTSD is as effective as weekly cognitive therapy and more effective than weekly supportive therapy
}

\section{Shannon Wiltsey Stirman}

National Center for PTSD, VA Boston Healthcare System, and Boston University, 150 S. Huntington Ave (116B-3), Boston, MA 02130, USA; shannon.wiltsey-stirman@va.gov

\section{WHAT IS ALREADY KNOWN ON THIS TOPIC?}

Cognitive behavioural therapy has been shown to be effective in treating post-traumatic stress disorder (PTSD) when delivered once or twice a week over several months. ${ }^{1}$ Cognitive therapies for PTSD are effective in reducing symptoms compared with self-help interventions with minimal therapist contact, but there are few comparisons to credible psychotherapy. ${ }^{12}$

\section{WHAT DOES THIS PAPER ADD?}

- Ehlers and colleagues' study compares an intensive cognitive therapy with a cognitive therapy delivered weekly. It is also one of the first comparisons between a cognitive therapy and supportive therapy for PTSD.

- The study demonstrates greater symptom reduction and less symptom worsening among patients who received intensive or standard cognitive therapy than those who received supportive therapy. It is also the first study to demonstrate that standard or intensive cognitive therapy results in large increases in quality of life as compared with the other interventions.

- Intensive cognitive therapy resulted in faster symptom reduction, good engagement and retention, and was well tolerated by patients.

\section{LIMITATIONS}

- As the study focused on traumatic events that occurred in adulthood, results might not generalise to the treatment of childhood trauma.

- More detailed information about the proportions of patients with different types of comorbid diagnoses, and how they fared in treatment would have been useful.

\section{WHAT NEXT IN RESEARCH?}

Insofar as a 7-day treatment may limit clinicians' abilities to guide patients in their efforts to reclaim their life, a long-term follow-up assessing quality of life and functioning would be useful. An examination of the treatment for patients with a broader array of index traumas (eg, childhood sexual abuse, military trauma) is an important next step. Finally, testing this treatment as a complement or follow-up to treatment for substance abuse could inform efforts to treat patients with co-occurring PTSD and substance use disorders.

\section{COULD THESE RESULTS CHANGE YOUR PRACTICES AND WHY?}

These results are unlikely to change my everyday cognitive-behavioral practice, as the schedule for intensive therapy would not be feasible for me or some of my patients. However, with advance planning and adjustments to schedules and caseload, I would provide intensive cognitive therapy occasionally for patients who have difficulty attending weekly sessions due to travel costs, their employment or other practical concerns. For some patients the benefit of more rapid symptom reduction and improvement in quality of life may be worth the more intensive short-term time commitment.

Competing interests None.

doi:10.1136/eb-2014-101857

\section{REFERENCES}

1. Ehlers A, Clark DM, Hackmann A, et al. A randomized controlled trial of cognitive therapy, a self-help booklet, and repeated assessments as early interventions for posttraumatic stress disorder. Arch Gen Psychiatry 2003;60:1024-32.

2. Resick PA, Galovski TE, O'Brien Uhlmansiek M, et al. A randomized clinical trial to dismantle components of cognitive processing therapy for posttraumatic stress disorder in female victims of interpersonal violence. J Consult Clin Psychol 2008;76:243-58.

ABSTRACT FROM: Ehlers A, Hackmann A, Grey N, et al. A randomized controlled trial of 7-day intensive and standard weekly cognitive therapy for PTSD and emotion-focused supportive therapy. Am J Psychiatry 2014;171:294-304.

Patients/participants One hundred and twenty-one adults (aged 18-65 years) meeting Diagnostic and Statistical Manual of Mental Disorders, fourth edition (DSM-IV) diagnostic criteria for chronic post-traumatic stress disorder (PTSD).

Setting National Health Service clinics in South London and Oxford, UK; 2003-2008.

Intervention Three different therapeutic interventions: a 7-day intensive cognitive therapy intervention $(n=30)$; 3 months of standard weekly cognitive therapy ( $n=31$ ); and 3 months of weekly, emotion-focused supportive therapy $(n=30)$. Standard cognitive therapy involved 12 weekly sessions over 3 months, followed by three optional monthly booster sessions. Intensive therapy followed the same protocol but involved up to $18 \mathrm{~h}$ of therapy delivered over 5-7 days.

Comparison The three interventions were compared with each other and to a waiting list control $(n=30)$ at 14 weeks (post-treatment). Further assessments of the three intervention groups were performed at 27 and 40 weeks.

Patient follow-up $95.9 \%$ Follow-up at 14 weeks; $90.1 \%$ across the intervention arms at 40 weeks.

Allocation Concealed.

Blinding Single blinded (assessors only).

\section{OUTCOMES}

PTSD recovery/loss of diagnosis (assessed using the ClinicianAdministered PTSD Scale (CAPS)) At 14 weeks, $73.3 \%$ of the intensive and $77.4 \%$ of the standard cognitive therapy groups had recovered compared with $43.3 \%$ of the supportive therapy group and $6.7 \%$ of the waiting list control group. Recovery rates were significantly higher in cognitive therapy groups compared with the supportive therapy group, and recovery rates with all three interventions were significantly higher than with waiting list control. At 40 weeks, recovery rates in the intensive and standard cognitive therapy groups $(66.7 \%$ and $74.2 \%$, respectively) were still significantly higher than the supportive therapy group $(40 \%)$. There was no significant difference in recovery rates between the intensive and cognitive therapy groups at either follow-up.

Assessor-rated total remission (assessed using CAPS) At 14 weeks, $46.7 \%$ of the intensive and $51.6 \%$ of the standard cognitive therapy groups had achieved remission, compared with $20 \%$ of the supportive therapy group and $3.3 \%$ of the waiting list control. At 40 weeks, remission rates in the intensive and standard cognitive therapy groups $(53.3 \%$ and $74.2 \%$, respectively) were still significantly higher than the supportive therapy group (26.7\%).

Self-reported total remission (assessed on the Posttraumatic Diagnostic Scale) Self-reported remission rates were significantly higher with intensive and standard cognitive therapy interventions, compared with supportive therapy at 14 weeks ( $56.7 \%$ and $64.5 \%$ vs $30 \%$ ) and 40 weeks (56.7\% and $58.1 \%$ vs $30 \%)$. 